RESEARCH PAPER

\title{
An evaluation of training in brief cognitive-behavioural therapy in a non-English-speaking region: experience from India
}

\author{
A. K. Gupta ${ }^{1}$ and H. Aman²
}

${ }^{1}$ Consultant Psychiatrist, Northumberland Tyne and Wear NHS Foundation Trust, Cherry Knowle Hospital Ryhope, Sunderland, UK, email Ryhope, Sunderland, UK, email
ArunKumar.Gupta@ntw.nhs.uk ${ }^{2}$ Registrar in the Child and Adolescent Psychiatry, Prudhoe Hospital, Prudhoe, Northumberland, UK

We acknowledge the support provided by Dr P. Psitholey and his colleagues at CSM Medial University, Lucknow. We also acknowledge the support of Dr R. Arjundas, Mr Thomas Reenes, Dr Steve Mooread Reeves, DrSteve Moorehed and Dr Suresh Joseph (Medical Director, NTW NHS Trust)
The authors wanted to learn whether it was possible to deliver cognitive-behavioural therapy (CBT) in a low-income country where English is neither the first nor the preferred spoken language and to evaluate the effectiveness of the training in terms of skills acquisition. Twenty participants attended a 3-day workshop on the technique. All had experience of communicating in Hindi with patients, although their medical training was in English. There is no manual for CBT in Hindi. Role-plays focused on basic CBT skills such as Socratic dialogue, the 'five area' approach, the use of the 'downward arrow', developing an automatic negative thought record and devising behavioural experiments, in Hindi. The findings suggested that it is feasible to train mental health professionals in CBT where English is not the first language.

\section{Background}

Growing evidence for the efficacy of cognitivebehavioural therapy (CBT) in the treatment of mental illnesses (Williams \& Garland, 2002) has stimulated the interest of psychiatrists in India. Over the course of 3 years, a series of 3-day workshops was organised by the first author at the Department of Psychiatry, Chhatrapati Sahuji Maharaja Medical University (CSMMU), previously known as King George's Medical College, Lucknow, India. This article focuses on outcome data from the fourth of these workshops. The first two workshops were at an introductory level, while the third focused on the use of behavioural experiments within a CBT context. (The results of these initial workshops have been presented at annual conferences of the British Association for Behavioural and Cognitive Psychotherapies and the Indian Psychiatry Society's Central Zone.)

The authors wanted to learn whether it was possible to deliver CBT in a low-income country where English is neither the first nor the preferred spoken language and to evaluate the effectiveness of the training in terms of skills acquisition.

\section{How delivering CBT training was different in India}

A commonly encountered barrier to initiating psychological therapies in non-English-speaking countries is the focus on biological/medical models of treatment on the part of both medical professionals and their patients. Training doctors in approaches that are new to them and their patients can be difficult. We distributed a report of a randomised controlled trail of CBT (King, 2002) to suggest to our psychiatric colleagues that CBT is an appropriate treatment for common mental health problems. An initial comment from one influential member of the local faculty was that CBT is appropriate for Western culture but may not work with Indian patients. This comment was turned into a question for the training programme and was presented as a behavioural experiment. The presumption was that if trainees are able to learn CBT skills, to use these with their patients and to get some good results in the first instance, it will be worthwhile developing a randomised controlled trial at a later stage for gathering strong scientific evidence in favour of the efficacy of CBT delivered to Indian patients in the local language.

Another barrier we faced was the hierarchical professional system within medicine in India (as is the case in many other low-income countries). When the workshop started, we noticed that there were four distinct groups: consultants, junior doctors, psychologists and medical students. We addressed this issue by ensuring a good mix of skills in each group when dividing them into smaller groups for skill practice, in order to give everyone a chance to participate fully in the training and to work as a team. There was naturally some apprehension among participants about this approach as it is difficult to break with tradition. But doing so helped the group to understand the concept of a collaborative approach.

The teaching environment and equipment were of a poorer standard than one would have in the UK and this necessitated a certain amount of improvisation. A 40-inch flat-screen television was attached to laptop via S-video cable and a home cinema surround-sound system was used for the audio output. We also utilised more roleplays in order to compensate for the lack of good presentation equipment. Role-plays focused on the demonstration of basic CBT skills by the first author, such as Socratic dialogue, discussing the 'five area' approach with the patient, the use of the 'downward arrow', developing an automatic negative thought record, devising behavioural experiments and so on, in Hindi. 
In order to help trainees consolidate and continue to practise skills learnt, some clinical supervision sessions were provided after the 3-day workshop, via telephone. Video-chat and email were also used, using the principles of distance learning. Trainees submitted therapy notes and case summaries for review. The supervision suggested that the trainees had been able to utilise CBT skills learnt during the workshop.

All participants had previous experience of communicating in Hindi with patients, although their medical training was in English. The first author worked with the participants during the workshop to agree Hindi equivalents (in the local dialect of the region) of common Socratic dialogue questions used in CBT, as there is no manual for CBT in Hindi. Unfortunately, the participants could not use rating scales when monitoring the results of CBT as there is a lack of standardised scales in Hindi.

\section{The workshop}

Twenty participants attended the course. The agenda for all three training days was agreed at the beginning of each session based on the learning objectives of the participants. Eight participants had attended previous workshops (in 2008 and 2009) and rest were new to the training (denoted 'repeat' and 'fresh', below). The fresh participants received introductory lectures from CSMMU faculty members beforehand, based on two books covering the basic principles of CBT (Hawton et al, 1998; Simmons \& Griffiths, 2009).

On day 1, the participants were asked to highlight their learning needs, which included understanding the 'five areas' model, assessment of patient suitability for CBT, the art of Socratic questioning and eliciting negative automatic thoughts. One of the participants volunteered to bring therapy notes (with the patient anonymous) to facilitate a discussion of the practical difficulties. The same participant was asked to role-play the patient, while the trainer (the first author) role-played the therapist and demonstrated the technique of Socratic dialogue to elicit negative automatic thought. A thought record was completed, based on the 'seven columns' technique as used by Greenberger \& Padesky (1995). The criteria used for the selection of patients for therapy were discussed (Blenkiron, 1999).

Day 2 started with a recap of day 1 . Based on feedback, an agenda for the day was agreed, which was to develop the skills of explaining the CBT model to patients and the rationale behind the behavioural experiments. A simple method was developed of explaining the CBT model to the patients in Hindi using an interactive vignette and Socratic dialogue.

Day 3 started with a recap of days 1 and 2 . An agenda was agreed to discuss supervision for trainee therapists and the practical aspects of behavioural experiments. Possible modifications to behavioural experiments based on cultural norms were also discussed and an experiment was devised based on a vignette brought by a trainee. The participants practised devising behavioural experiments in groups of three or four and the first author supervised the skill practice.

\section{Assessment}

The baseline knowledge of all the participants was assessed before the workshop, using a questionnaire designed for participants to self-rate their core CBT skills. It covered seven areas: activity scheduling; the use of the positive data log; Socratic dialogue; core belief; negative automatic thought; formulation; and all-or-nothing thinking.

Participants were asked to complete the questionnaire again after the workshop. There was no formal measure of competency following the

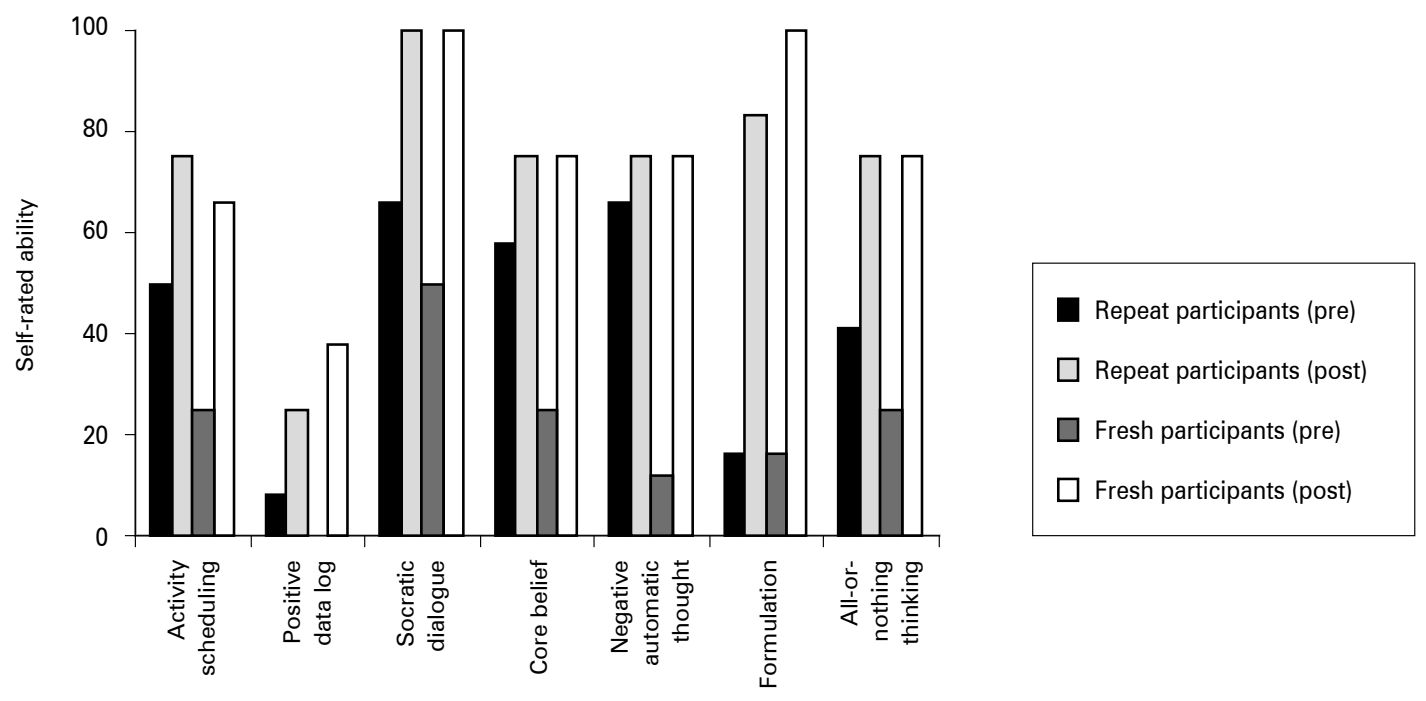

Fig. 1

Comparison of the self-evaluated skill ratings of the fresh and repeat participants before and after the workshop. 


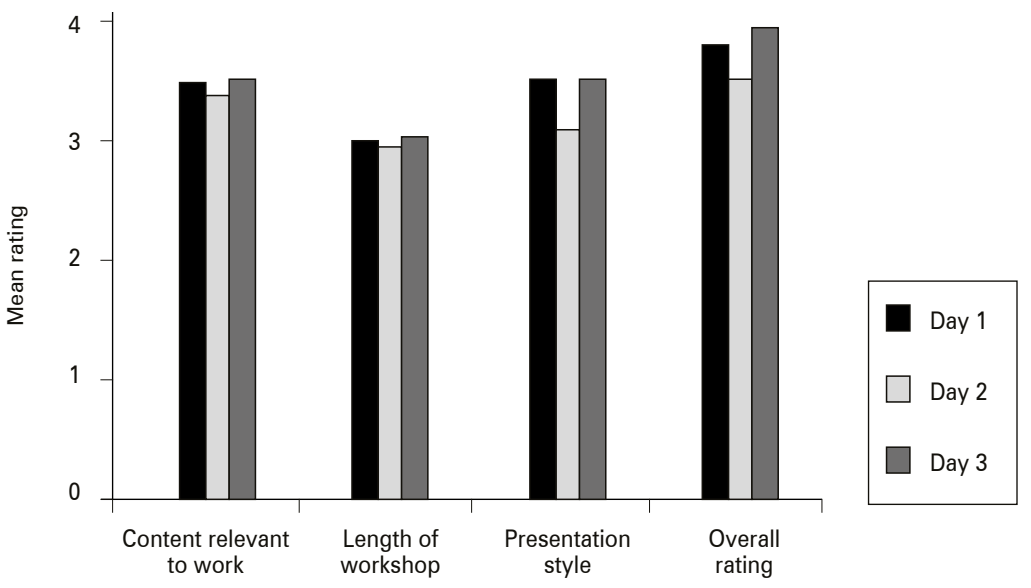

Fig. 2

Feedback on each of the 3 days of the workshop

course owing to a lack of facilities such as videoequipment and difficulty in gaining consent from patients because of issues of stigma.

All the trainees were asked to provide feedback at the end of each day on four different aspects of the workshop: content relevant to work, length of workshop, presentation style and overall rating. These were scored on a scale of $0-4$ : 0 , poor; 1 , fair; 2, good; 3, very good; 4, excellent.

All the data collected were analysed using SPSS. Means and standard errors (s.e.) were calculated and significance was determined using paired $t$-tests.

\section{Results and discussion}

We found that all participants (both repeat and fresh) improved after training (mean score on the self-assessment questionnaire 75.29 , s.e. $=5.95$ ) compared with baseline (mean 32.71 , s.e. $=5.92$ ) $(t(13)=-6.899, P<0.001, r=0.886)$ (see Fig. 1).

In the fresh participants group a skill level of $100 \%$ was noted for Socratic dialogue and formulation. There was no difference observed in either cohort of trainees in their understanding of core belief and negative automatic thoughts, which reached satisfactory levels (around 70\%). Skills like activity scheduling, core belief, negative automatic thoughts and all-or-nothing thinking showed some improvement (to around $75 \%$ ). There was poor understanding of the use of the positive data $\log$ $(25 \%)$ among all the trainees and this area needs to be addressed in future workshops.

The trainees' feedback is summarised in Fig. 2.

\section{Areas for improvement/limitations of the study}

- There is a lack of standardised rating scales in Hindi for the measurement of mental illness.
The first author has started work on one such measure.

- The questionnaire we used was from an unpublished small-scale research project and so its validity is questionable. Also, it is based on self-evaluation and lacks objective assessment.

- Accuracy of measurement of skills development would have been improved if formal assessment measures were used and this needs further consideration for future training.

- The lack of suitable teaching aids such as audioand video-equipment needs to be addressed.

- As the participants had different levels of theoretical knowledge at the beginning of training it was difficult to compare the groups to measure the outcome of training.

- Future work is now focusing on the development of rating scales in Hindi, video-recording of therapy sessions, the use of formal assessment measures and the development of randomised controlled trials for evaluating the efficacy of CBT in an Indian population.

\section{Conclusion}

This initial study would suggest that it is feasible to train mental health professionals in CBT in India and possibly other countries where English is not the first language. As this was a small project with a number of methodological constraints, the evidence cannot be seen as conclusive. In the context of training mental health professionals, the central indicator of an effective training programme would be an improvement in client care and clinical outcomes (Kirkpatrick, 1979). There is therefore a need to carry out more robust work in recruiting more patients and carrying out systematised research.

\section{References}

Blenkiron, P. (1999) Who is suitable for cognitive behaviour therapy? Journal of the Royal Society of Medicine, 92, 222-229.

Greenberger, D. \& Padesky, CA. (1995) Mind over Mood. Guilford Press.

Hawton, K., Salkovskis P.M., Kirk, J., et al (1998) Cognitive Behaviour Therapy for Psychiatric Problems: A Practical Guide. Oxford University Press.

King, M. (2002) Effectiveness of teaching general practitioners skills in brief cognitive behaviour therapy to treat patients with depression: randomised controlled trial. BMJ, 324, 947.

Kirkpatrick, D. L. (1979) Techniques for evaluating training programs. Training and Development Journal, 33, 78-92.

Simmons, J. \& Griffiths, R. (2009) CBT for Beginners. Sage.

Williams, C. \& Garland, A. (2002) Identifying and challenging unhelpful thinking. Advances in Psychiatric Treatment, 8, 377-386. 